\title{
Effects of insemination and blood-feeding on locomotor activity of Aedes albopictus and Aedes aegypti (Diptera: Culicidae) females under laboratory conditions
}

Tamara Nunes Lima-Camara ${ }^{1,2,3}$, José Bento Pereira Lima ${ }^{4,5,6}$, Rafaela Vieira Bruno $2,6^{*}$ and Alexandre Afranio Peixoto $2,6^{\wedge}$

\begin{abstract}
Background: Dengue is an arbovirus disease transmitted by two Aedes mosquitoes: Ae. aegypti and Ae. albopictus. Virgin females of these two species generally show a bimodal and diurnal pattern of activity, with early morning and late afternoon peaks. Although some studies on the flight activity of virgin, inseminated and blood-fed Ae. aegypti females have been carried out under laboratory conditions, little is known about the effects of such physiological states on the locomotor activity of Ae. albopictus and Ae. aegypti females. The aim of this study was to analyze, under laboratory conditions, the effects of insemination and blood-feeding on the locomotor activity of Ae. albopictus and Ae. aegypti females under LD 12:12, at $25^{\circ} \mathrm{C}$.

Methods: Both Ae. albopictus and Ae. aegypti females were obtained from established laboratory colonies. Control groups were represented by virgin/unfed Ae. albopictus and Ae. aegypti females. Experiments were conducted under laboratory conditions, using an activity monitor that registers individual activity every thirty minutes.

Results: Virgin/unfed Ae. albopictus and Ae. aegypti females showed a diurnal and bimodal pattern of locomotor activity, with peaks at early morning and late afternoon. Insemination and blood-feeding significantly decreased the locomotor activity of Ae. aegypti females, but inseminated/blood-fed Ae. aegypti and Ae. albopictus females showed a similar significant decrease on the locomotor activity compared to virgin/unfed females.

Conclusions: This study is the first demonstration of the effects of insemination and blood-feeding on the locomotor activity of Ae. albopictus and Ae. aegypti females under artificial conditions. Data suggest that Ae. albopictus and Ae. aegypti females respond in different ways to physiological status changes and such divergence between these two dengue vectors, associated with several ecological differences, could be related to the greater dengue vectorial capacity of Ae. aegypti in Americas in comparison to Ae. albopictus.
\end{abstract}

Keywords: Aedes albopictus, Aedes aegypti, Physiology, Locomotor activity, Laboratory

\footnotetext{
* Correspondence: rafaelav@ioc.fiocruz.br

Deceased

2Laboratório de Biologia Molecular de Insetos, Instituto Oswaldo Cruz, IOCFIOCRUZ, Av. Brazil, 4365, Manguinhos, Rio de Janeiro CEP 21040-360, Brazil

${ }^{6}$ Instituto Nacional de Ciência e Tecnologia em Entomologia Molecular (INCT-EM)/CNPq, Rio de Janeiro, Brazil

Full list of author information is available at the end of the article
}

\section{Biomed Central}

(C) 2014 Lima-Camara et al.; licensee BioMed Central Ltd. This is an Open Access article distributed under the terms of the Creative Commons Attribution License (http://creativecommons.org/licenses/by/4.0), which permits unrestricted use, distribution, and reproduction in any medium, provided the original work is properly credited. The Creative Commons Public Domain Dedication waiver (http://creativecommons.org/publicdomain/zero/1.0/) applies to the data made available in this article, unless otherwise stated. 


\section{Background}

Dengue is an arbovirus disease predicted to be ubiquitous throughout the tropics and it is strongly influenced by temperature, rainfall and high urbanization. Recently, 96 million apparent infections and 293 million unapparent infections of dengue cases were estimated in the world per year [1]. Aedes (Stegomyia) aegypti (Linnaeus 1762) is considered the main dengue vector in the world and has a wide distribution in tropical countries. Aedes (Stegomyia) albopictus (Skuse 1994) originated in Asia, where it can play an important role as a dengue vector, and also has a wide distribution in tropical as well as temperate countries [2]. In Brazil, as well as in a few countries, Ae. albopictus is considered a potential vector of dengue and other arboviruses, such as yellow fever and Chikungunyia [3-5].

Daily rhythms in behaviours, such as flight, mating, blood-feeding and oviposition are observed in mosquitoes, which restrict their activities to specific hours of the day. This is due to an endogenous circadian clock that can be synchronized to external cues, such as light, temperature and food, among others [6]. In insect vectors, the relationship between the circadian clock and the different behaviours are particularly important to their vectorial capacity. Besides, the different physiological states of the individuals can modulate the way they respond to several stimuli. For instance, in the sandfly Lutzomyia longipalpis, the main vector of American Visceral Leishmaniasis, blood-fed females present a significant decrease of their activity pattern in comparison to the unfed ones [7]. In Anopheles gambiae, the main malaria vector in Africa, circadian rhythmicity drives inseminated females to engage in hostseeking flight characterised by a broad peak of activity throughout the night [8], in contrast to the bimodal activity of virgin females seeking mating swarms [9]. When mosquitoes are unfed, the host-seeking behaviour can be modified by host-associated stimuli [10].

According to their locomotor activity pattern, Ae. aegypti and Ae. albopictus are considered diurnal vectors, with more activity during the early morning and the late afternoon under natural conditions $[2,11,12]$. In general, virgin Ae. aegypti and Ae. albopictus males and females copulate two or three days after emergence and inseminated females seek a vertebrate host to blood-feed and develop eggs [13-15]. Insemination and blood-feeding are two factors that might affect the behaviour of Ae. aegypti and Ae. albopictus females. In fact, the accessory glands of Aedes male mosquitoes produce substances that are transferred to females during mating that can influence their circadian rhythmicity and also alter some aspects of female physiology and behaviour, such as mating, preoviposition and host-seeking behaviours $[16,17]$. Under laboratory conditions, the flight activity of virgin, inseminated and bloodfed Ae. aegypti females has been evaluated [18]. The author reported significant differences on the total flight activity of the specimens and showed that it is important to analyze the pattern of activity of virgin females as well as inseminated and blood-fed ones [18].

Although some studies have been done on the flight activity of Ae. aegypti females in different physiological states [19], little is known about the locomotor activity of inseminated and/or blood-fed Ae. albopictus and Ae. aegypti females in artificial conditions. Thus, the aim of this study was to evaluate, under laboratory conditions, the effect of insemination and blood-feeding on the locomotor activity of Ae. albopictus and Ae. aegypti females.

\section{Methods}

\section{Mosquito rearing}

Ae. aegypti Rockefeller strain adult mosquitoes were obtained from colonies of LAFICAVE since 1986 (Laboratório de Fisiologia e Controle de Artrópodes Vetores, Fiocruz, Rio de Janeiro, Brazil), whereas Ae. albopictus adults were obtained from colonies established in LATHEMA (Laboratório de Transmissores de Hematozoários, Fiocruz, Rio de Janeiro, Brazil). Immature forms were raised in trays with tap water and fish food (Tetramin ${ }^{\oplus}$ ) [2], under LD 12:12, at $25^{\circ} \mathrm{C}$. Pupae of both species were isolated individually to ensure virgin males and females after emergence. For experiments with inseminated females, approximately 50 three-days old females were placed into a cage with approximately 100 males of the same age, for 24 hours.

For experiments with blood-fed females, 4-5-day old females were fed on anesthetized Guinea pig (ketamine: xylazine $80-120: 10-16 \mathrm{mg} / \mathrm{kg}$ ) for 2 hours, according to institutional procedures, oriented by the national guideline 'the Brazilian legal framework on the scientific use of animals' [20]. Locomotor activity analysis started at 24 hours after insemination and 2 hours after blood feeding.

Cotton soaked in a $15 \%$ sucrose solution was continuously available for all mosquitoes as a sugar source, except for experiments with blood-fed females, when sucrose solution was taken out 24 hours before blood-feeding. All cages with Aedes adults were placed inside the Precision Scientific Incubator Mod. 818, under same experimental photoperiod, humidity and temperature conditions as the raising phase. Spermathecae of all inseminated females were dissected at the end of locomotor activity experiments to ensure insemination.

\section{Analysis of locomotor activity}

Locomotor activity pattern of virgin/unfed (control group), inseminated/unfed, virgin/blood-fed and inseminated/ blood-fed Ae. aegypti females was evaluated with a Locomotor Activity Monitor (TriKinetics), first developed for Drosophila. Locomotor activity is one of the 24 hour scale rhythms that can be monitored over many 
days. Adult females are placed into small glass tubes and those are placed into special trays equipped with infrared light and detectors. By introducing the infrared beam into the path of the mosquito, a computer counts every time the beam is broken, giving a measure of the pattern of activity and rest of the mosquito [21].

Four up to five - day -old adults were individually placed in glass tubes $(10 \mathrm{~mm} \times 7 \mathrm{~cm})$ with a cotton plug soaked in $15 \%$ sucrose solution and these tubes placed in a Locomotor Activity Monitor inside a Precision Scientific Incubator Mod. 818 under a constant temperature of $25^{\circ} \mathrm{C}$ and a photoperiod of 12 hours of light and 12 hours of dark (LD 12:12), as previously described [22-24]. All locomotor activity experiments started with 32 individuals.

For every mosquito, 48 data points representing the total locomotor activity of 30 minute intervals were obtained for every day of monitoring. All females that survived the first 3 days of experiment were analyzed. We calculated the Williams mean (Wm) as an estimate of the central tendency of activity during each time interval. The Williams mean is a modified geometric mean that accommodates frequent zero values [25].

\section{Statistical analysis}

For statistical analyses, we first tested the normality of all data using the Kolmogorov-Smirnov test. Since the normality assumptions were satisfied, we used the parametric independent samples T-test.

The daily locomotor activity was summarized by seven indices: (1) the total activity mean; (2) the diurnal activity mean; (3) the diurnal activity without lights-on mean, i.e., the mean activity during the photophase except for the first 30 minutes, which corresponds to the morning peak, (4) the lights-on mean, which corresponds to the first 30 minutes just after the lights-on, (5) the nocturnal activity mean, (6) the nocturnal activity without lightsoff mean, i.e., the mean activity during the scotophase except for the first 30 minutes, which corresponds to the evening peak and (7) the lights-off mean, which corresponds to the first 30 minutes just after the lights-off.

\section{Results}

Figure 1 shows the locomotor activity of inseminated/unfed Ae. aegypti (A; red line) and Ae. albopictus (B; blue line) females compared to the respective control groups (virgin/ unfed females - black lines) under two days of LD 12:12, at $25^{\circ} \mathrm{C}$. First day in the graph represents the next day after blood-feeding $(24 \mathrm{~h})$ and two days after insemination (48 hours).

In general, Ae. aegypti (Figure 1A) and Ae. albopictus (Figure 1B) control females (virgin/unfed) showed a diurnal and bimodal pattern of activity, with peaks at lights-on and lights-off, and more activity at the end of photophase, in a characteristic nocturnal anticipation. Inseminated/unfed
Ae. aegypti (Figure 1A; red line) and Ae. albopictus females (Figure 1B; blue line) also showed a diurnal and bimodal pattern of activity, with smaller peaks at lights-on and higher peak at lights-off compared to the control group (Figures 1A and B; black lines). Inseminated/unfed Ae. aegypti females showed significant decreases in the diurnal mean $(\mathrm{t}=2.567 ; \mathrm{df}=62 ; \mathrm{p}=0.013)$, diurnal mean without lights-on $(\mathrm{t}=2.417 ; \mathrm{df}=62 ; \mathrm{p}=0.019)$, lights-on mean $(\mathrm{t}=2.195 ; \mathrm{df}=50.549 ; \mathrm{p}=0.033)$ and lights-off mean $(\mathrm{t}=-2.305 ; \mathrm{df}=55.327 ; \mathrm{p}=0.025)$ compared to control group. Nevertheless, no significant decrease was observed in any of the parameters evaluated for inseminated/unfed Ae. albopictus females compared to virgin/unfed Ae. albopictus females.

Figure 2 shows the locomotor activity of virgin/blood-fed Ae. aegypti (A; red line) and Ae. albopictus (B; blue line) females compared to the respective control groups (virgin/unfed females - black lines) under two days of LD $12: 12$, at $25^{\circ} \mathrm{C}$. First day in the graph represents the next day after blood-feeding $(24 \mathrm{~h})$ and two days after insemination (48 hours). Ae. aegypti (Figure 2A) and Ae. albopictus (Figure $2 \mathrm{~B}$ ) control females (virgin/unfed; black lines) showed a diurnal and bimodal pattern of activity, with peaks at lights-on and lights-off, and more activity at the end of photophase (Figures 2A and B). Virgin/blood-fed Ae. aegypti (Figure 2A; red line) and Ae. albopictus females (Figure 2B; blue line) also showed a diurnal and bimodal pattern of activity, but lights-on and lights-off peaks of $A e$. aegypti control females were higher than virgin/blood-fed Ae. aegypti females (Figure 2A), whereas virgin/blood-fed Ae. albopictus females showed a smaller lights-on peak and a higher lights-off peak compared to virgin/unfed $A e$. albopictus females (Figure 2B).

Compared to virgin/unfed Ae. aegypti females, virgin/ blood-fed Ae. aegypti females showed a significant decrease in the mean activity of two days $(\mathrm{t}=2.553 ; \mathrm{df}=59 ; \mathrm{p}=$ $0.013)$, diurnal mean $(\mathrm{t}=4.239 ; \mathrm{df}=59 ; \mathrm{p}<0.001)$, diurnal mean without lights-on $(\mathrm{t}=3.931 ; \mathrm{df}=59 ; \mathrm{p}<0.001)$ and lights-on mean $(t=3.761 ; d f=59 ; p<0.001)$. On the other hand, except for the lights-on mean $(\mathrm{t}=2.098 ; \mathrm{df}=54 ; \mathrm{p}=$ 0.041), similar to inseminated/unfed Ae. albopictus females, virgin/blood-fed Ae. albopictus females showed no significant decrease in any of the parameters evaluated when compared to virgin/unfed females.

As verified in control females of Figures 1 and 2, the locomotor activity of control Ae. aegypti and Ae. albopictus females (virgin/unfed) (Figure 3) was also diurnal, with more activity at the end of photophase and bimodal, with peaks at lights-on and lights-off, generally higher than inseminated/blood-fed Ae. aegypti (Figure 3A; red line) and Ae. albopictus (Figure 3B; blue line) females. Inseminated/ blood-fed Ae. aegypti (Figure 3A; red line) and Ae. albopictus (Figure 3B; blue line) females showed very low activity in the two tested days, showing almost no lights-on peak 

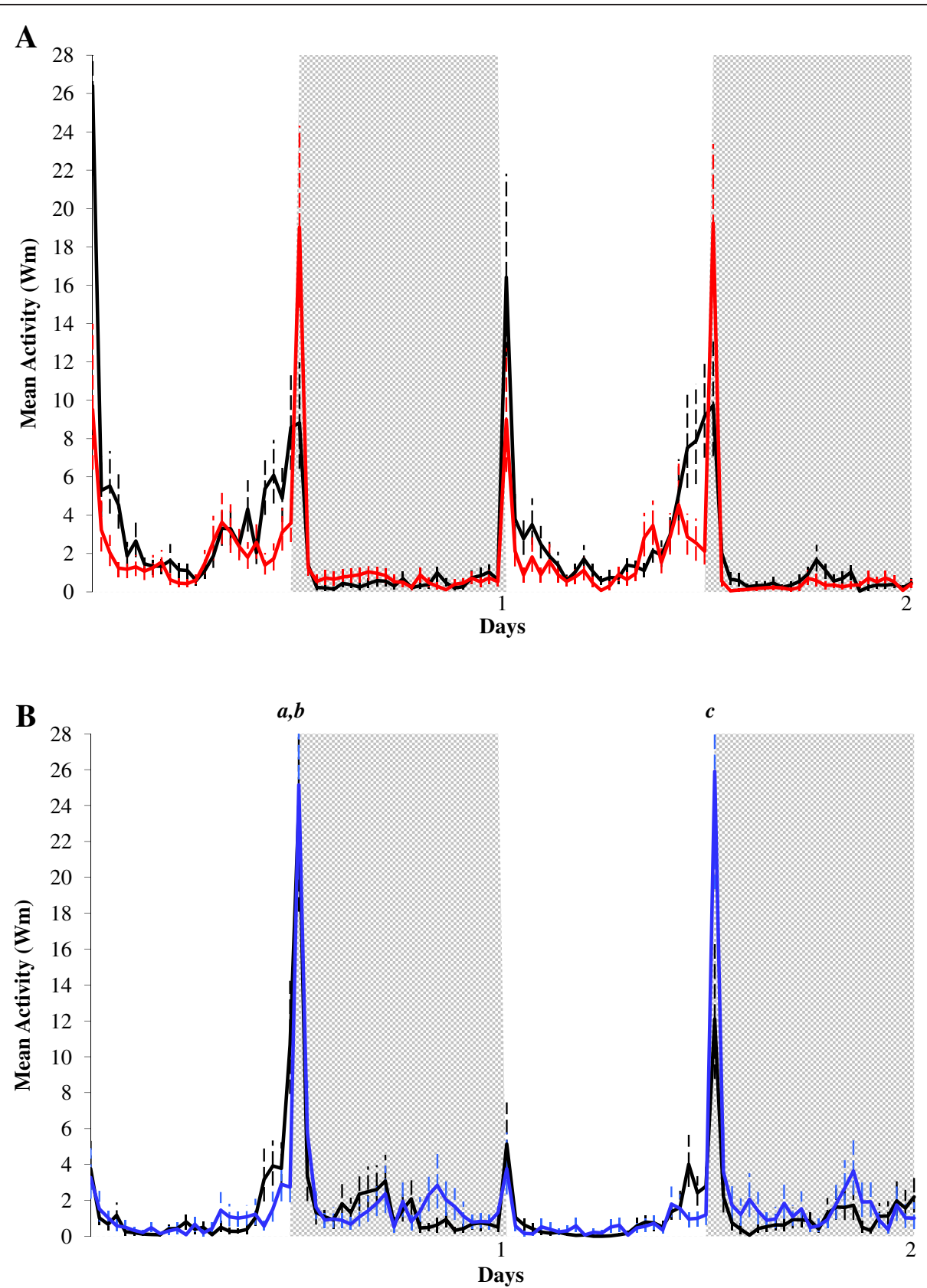

Figure 1 Effects of insemination on the locomotor activity of Aedes aegypti (A) and Aedes albopictus (B). Locomotor activity of virgin/ unfed (black line; $n=32$ for Ae. aegypti and $n=24$ for Ae. albopictus) and of inseminated/unfed (red line) Ae. aegypti females $(\mathbf{A} ; n=32)$ and (blue line) Ae. albopictus females $(\mathbf{B} ; \mathrm{n}=24)$ exposed to two days of $L D$ 12:12, at $25^{\circ} \mathrm{C}$. First and second days represent, respectively, 48 and 72 hours after insemination. White column represents the photophase and grey column the scothophase. Some reading time points exceeding the scale are indicated with letters, and their values are as follows: $a$ (virgin/unfed) $=23.97(+7.69) ; b$ (inseminated/unfed) $=25.15(+6.61) ; c=25.90(+8.68)$.

and activity in the end of photophase. The lights-off peaks were higher in both inseminated/blood-fed $A e$. aegypti and Ae. albopictus females compared to control females (Figure 3). Significant decreases in the total activity mean $(\mathrm{t}=8.508 ; \mathrm{df}=48.196 ; \mathrm{p}<0.001)$, diurnal mean $(\mathrm{t}=9.705 ; \mathrm{df}=48.790 ; \mathrm{p}<0.001)$, diurnal mean without lights-on $(\mathrm{t}=8.930 ; \mathrm{df}=48.498 ; \mathrm{p}<0.001)$ and lights-on mean $(\mathrm{t}=11.479 ; \mathrm{df}=54 ; \mathrm{p}<0.001)$ were verified for inseminated/blood-fed Ae. aegypti females compared to the virgin/unfed Ae. aegypti females. Similar significant decreases were reported for the total activity mean $(\mathrm{t}=$ 3.312; $\mathrm{df}=43.298 ; \mathrm{p}=0.002)$, diurnal mean $(\mathrm{t}=4.123$; $\mathrm{df}=52 ; \mathrm{p}<0.001)$, diurnal mean without lights-on $(\mathrm{t}=$ $3.657 ; \mathrm{df}=52 ; \mathrm{p}=0.001)$ and lights-on mean $(\mathrm{t}=4.652$; 

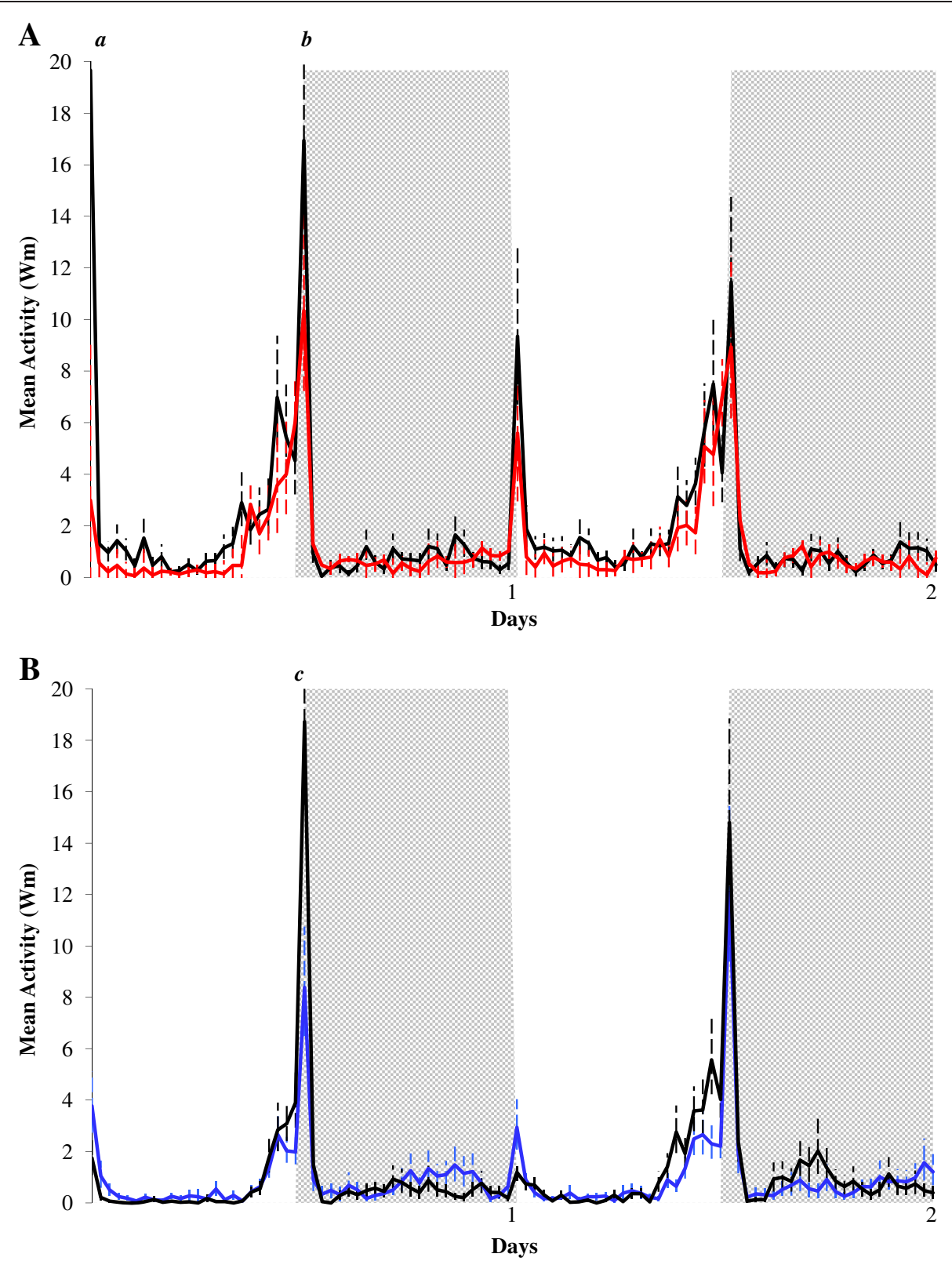

Figure 2 Effects of blood-feeding on the locomotor activity of Aedes aegypti (A) and Aedes albopictus (B). Locomotor activity of virgin/ unfed (black line; $n=32$ for Ae. aegypti and $n=28$ for Ae. albopictus) and of virgin/blood-fed (red line) Ae. aegypti females $(\mathbf{A} ; n=29)$ and (blue line) Ae. albopictus females $(\mathbf{B} ; n=28)$ exposed to two days of $L D 12: 12$, at $25^{\circ} \mathrm{C}$. First and second days represent, respectively, 24 and 48 hours after blood-feeding. White column represents the photophase and grey column the scothophase. Some reading time points exceeding the scale are indicated with letters, and their values are as follows: $a=19.66(+6.15) ; b=16.93(+3.77) ; c=18.72(+3.86)$.

$\mathrm{df}=52 ; \mathrm{p}<0.001)$ were verified for inseminated/bloodfed Ae. albopictus females compared to the virgin/unfed Ae. albopictus females.

\section{Discussion}

Circadian rhythms have been extensively studied in insects due to their importance in regulating many behaviours, especially in vector species. Unlike the model Drosophila melanogaster, which comprises behavioural and genetic evidences of the regulation of these rhythms, only recently studies on insect vectors are shedding light on this issue.

In Anopheles gambiae, an inhibition in blood feeding was observed in females with reduced expression of clock genes via RNAi [26], corroborating the idea that clock genes regulate blood feeding. Thus, further transcriptome studies showed classes of genes regulated by the circadian clock, including detoxification, redox and metabolism genes [27]. In the same way, Lutzomyia longipalpis also present a 

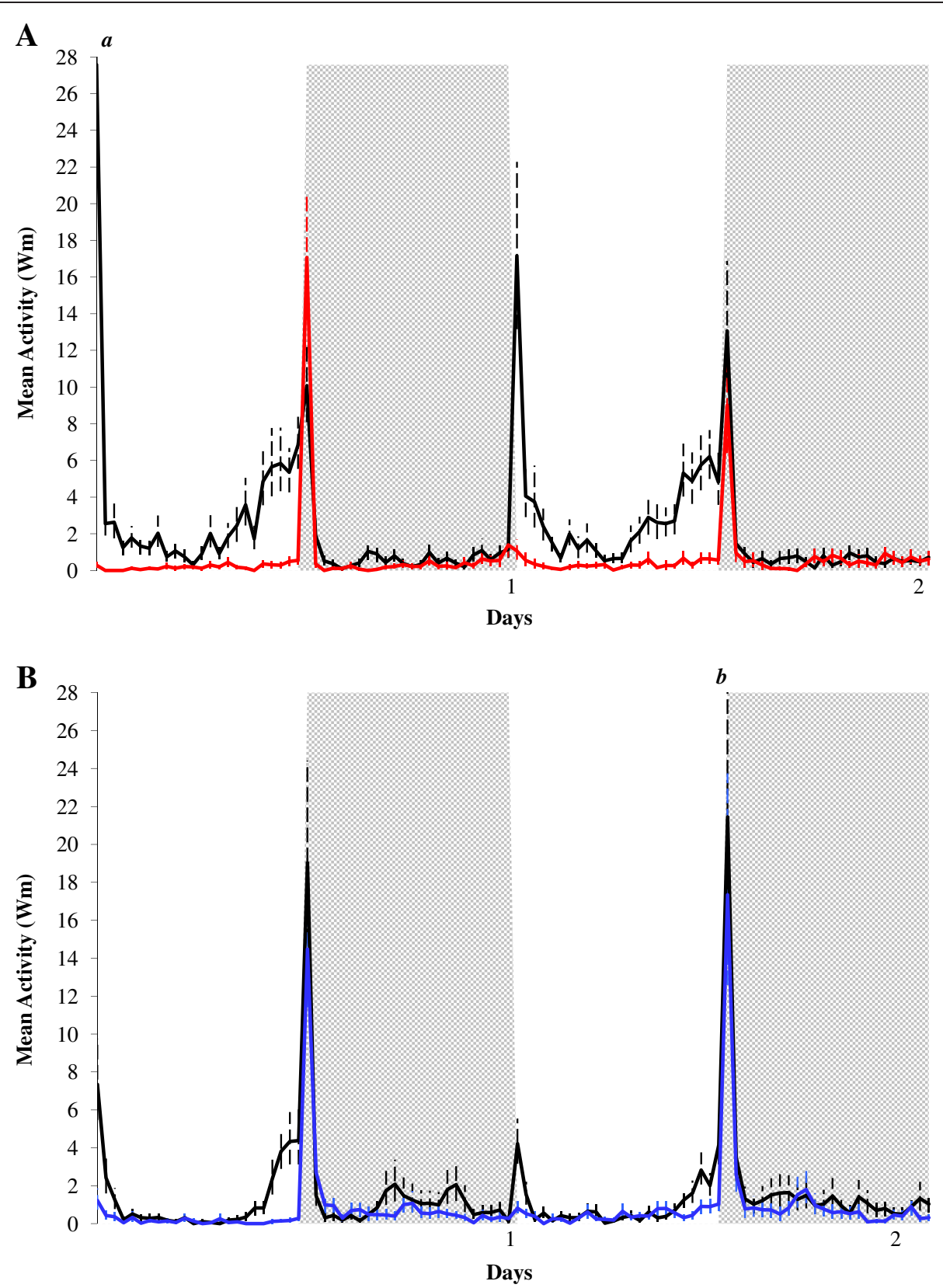

Figure 3 Effects of insemination and blood-feeding on the locomotor activity of Aedes aegypti (A) and Aedes albopictus (B). Locomotor activity of virgin/unfed (black line; $n=31$ for Ae. aegypti and $n=28$ for Ae. albopictus) and of inseminated/blood-fed (red line) Ae. aegypti females $(\mathbf{A} ; \mathrm{n}=25)$ and (blue line) Ae. albopictus females $(\mathbf{B} ; \mathrm{n}=26)$ exposed to two days of $L D 12: 12$ at $25^{\circ} \mathrm{C}$. First and second days represent, respectively, 48 and 72 hours after insemination and 24 and 48 hours after blood-feeding. White column represents the photophase and grey column the scothophase. Some reading time points exceeding the scale are indicated with letters, and their values are as follows: $a=27.57$ $(+6.81) ; b=21.44(+6.79)$.

robust decrease in the amplitude of the locomotor activity in blood fed females [7].

Bimodal and diurnal rhythms have already been reported for Ae. aegypti and Ae. albopictus females when testing flight, sugar-feeding and host-seeking activities under laboratory conditions $[18,28,29]$. In addition, under laboratory conditions, it has been reported that parasite-vector interactions, such as dengue virus- Ae. aegypti infection, as well as genetic mutations which denote insecticide resistance by the vector do not change the pattern of activity of $A e$. aegypti, but increase the locomotor activity of females $[24,30]$. Although it has been reported that physiological states, such as insemination and blood-feeding, affect the flight activity of Ae. aegypti females [18], it has not been 
evaluated up to now how drastic changes in these physiological states could affect the locomotor activity pattern of Ae. aegypti and Ae. albopictus females.

In general, Ae. aegypti females show great alterations in their pattern of activity when they are inseminated, because male accessory gland (MAG) produces some peptides and other elements that are transferred to females during copulation and modulate their host-seeking behaviour [16,17]. It is also known that Aedes albopictus MAG is able to decrease the diurnal locomotor activity of $A e$. aegypti females in a similar way to Ae. aegypti MAG, making them refractory to mating with conspecific males [31]. This refractoriness may lead to a reduction in the vectorial capacity of both species. Such modifications have also been described in other mosquito species, including $A n$. gambiae [8,9] and An. stephensi [32].

In this study, we observed that inseminated/unfed $A e$. aegypti females showed a significant decrease in the locomotor activity compared to virgin/unfed females, whereas inseminated/unfed Ae. albopictus females did not show significant decrease in the locomotor activity compared to the control group. This could be explained by the ecological needs of each species. It is well known that $A e$. aegypti has a close association with human houses, where they mate and blood-feed $[18,19]$. Thus, after mating, $A e$. aegypti females do not need to be active for a long period to find a vertebrate and blood-feed [33]. On the other hand, Ae. albopictus is a more sylvatic species [2], needing to be active for longer periods to mate and, then, seek a vertebrate to blood-feed. A similar decrease in flight activity of inseminated/unfed Ae. aegypti females has been reported under laboratory conditions [18], but no previous data have shown the effect of insemination on the activity of Aedes albopictus females.

A fully engorged female is much heavier than an unfed one and it has been reported that abdominal distention by blood feeding or developing ovaries can inhibit the activity of host-seeking of Ae. aegypti females [34,35]. Thus, until eggs are fully developed and ready to be laid, we expect a decrease in locomotor activity in both engorged Aedes females, but it was only observed in Ae. aegypti females. Once more, the explanation for this difference could rely on the ecology of each species, as mentioned above. In addition, under laboratory conditions, it has been demonstrated that insemination and blood-feeding can affect the circadian expression of specific genes involved in circadian clocks and, consequently, in circadian rhythms of Ae aegypti and other hematophagous vectors $[7,35]$. In the opposite way, when clock gene expression is reduced, the blood feeding is inhibited, indicating that not only the clock genes expression per se but also the clock controlled genes (ccg's) are fundamental for the blood feeding behaviour [26]. The circadian expression of such specific genes that control circadian rhythms in inseminated and blood-fed Ae. albopictus females deserves further investigations.

Inseminated/blood-fed Ae. aegypti and Ae. albopictus females showed similar decreases in locomotion compared to virgin/unfed Ae. aegypti and Ae. albopictus. Under natural conditions, 2-3 days after being inseminated, Ae. aegypti and Ae. albopictus females seek a vertebrate host to blood-feed $[14,15]$. Blood digestion triggers ovarian development and females are considered gravid and ready to oviposit three days later [36]. Thus, we can expect low activities of inseminated/blood-fed Ae. aegypti and Ae. albopictus females in the first two days since eggs are already being developed to be oviposited about three days later.

Except for the lights-off mean of inseminated/unfed $A e$. aegypti females, neither insemination nor blood-feeding significantly affected the nocturnal mean, the nocturnal mean without lights-off and the lights-off mean of both species compared to the virgin/unfed females. This data can be explained by the frequent diurnal behaviour shown by Ae. aegypti and Ae. albopictus under natural conditions, flying, mating and blood-feeding preferentially during the early morning and late afternoon hours [37].

\section{Conclusions}

This study is the first demonstration of the effects of insemination and blood-feeding on the locomotor activity of Ae. albopictus and Ae. aegypti females under artificial conditions. Since we have compared the behaviour of Ae. albopictus and Ae. aegypti females from laboratory colonies, it would be helpful if insemination and blood-feeding effects could also be studied with F1 or F2 generations of these mosquitoes. Nevertheless, this study observed several differences between the effects of insemination and bloodfeeding on these two dengue vector females that, associated with some ecological differences between both species, could be related to the better dengue vectorial capacity of Ae. aegypti in Americas in comparison to Ae. albopictus. Thus, further studies of insemination and blood-feeding effects on Ae. albopictus and Ae. aegypti females are necessary to fully understand the biology and behaviour of these two important dengue vectors.

\section{Competing interests}

The authors declare that they have no competing interests.

\section{Authors' contributions}

TNLC and AAP designed the study. JBPL raised the mosquito larvae and carried out the blood-feeding of females. TNLC, AAP and RVB analyzed and interpreted all locomotor activity data. AAP supervised all laboratory experiments, analysis of the data and interpretation of results. TNLC and RVB were involved in drafting the manuscript. All authors approved the final version of the manuscript.

\section{Acknowledgment}

This work is dedicated to the memory of Alexandre Afranio Peixoto, an outstanding friend and scientist. This work was funded by HHMI, Fiocruz, FAPERJ and CNPq. 


\section{Author details}

${ }^{1}$ Programa de Computação Científica - PROCC. Fundação Oswaldo Cruz/ FIOCRUZ, Av. Brazil, 4365, Manguinhos, Rio de Janeiro CEP 21040-360, Brazil. ${ }^{2}$ Laboratório de Biologia Molecular de Insetos, Instituto Oswaldo Cruz, IOCFIOCRUZ, Av. Brazil, 4365, Manguinhos, Rio de Janeiro CEP 21040-360, Brazil. ${ }^{3}$ Departamento de Epidemiologia da Faculdade de Saúde Pública da USP, Arnaldo, 715, Cerqueira César, São Paulo CEP - 01246-904, Brazil. Laboratório de Fisiologia e Controle de Artrópodes Vetores, Instituto Oswaldo Cruz, IOCFIOCRUZ, Av. Brazil, 4365, Manguinhos, Rio de Janeiro CEP 21040-360, Brazil. ${ }^{5}$ IBEX - Instituto de Biologia do Exército, Rua Francisco Manuel, 102, Benfica, Rio de Janeiro CEP 20911-270, Brazil. ${ }^{6}$ Instituto Nacional de Ciência e Tecnologia em Entomologia Molecular (INCT-EM)/CNPq, Rio de Janeiro, Brazil.

Received: 14 February 2014 Accepted: 26 June 2014 Published: 2 July 2014

\section{References}

1. Bhatt SP, Gething W, Brady OJ, Messina JP, Farlow AW, Moyes CL, Drake JM, Brownstein JS, Hoen AG, Sankoh O, Myers MF, George DB, Jaenisch T, Wint GR, Simmons CP, Scott TW, Farrar JJ, Hay SI: The global distribution and burden of dengue. Nature 2013, 496:504-507.

2. Consoli RAGB, Lourenco-de-Oliveira R: Principais mosquitos de importância sanitária no Brasil. Rio de Janeiro: Fiocruz; 1994

3. Gratz NG: Critical review of the vector status of Aedes albopictus. Med Vet Entomol 2004, 18:215-227.

4. Lounibos LP: Competitive displacement and reduction. J Am Mosq Control Assoc 2007, 23(Suppl 2):276-282.

5. Vega-Rúa A, Zouache K, Girod R, Failloux AB, Lourenço-de-Oliveira R: High level of vector competence of Aedes aegypti and Aedes albopictus from ten American countries as a crucial factor in the spread of Chikungunya virus. J Virol 2014, 88:6294-6306.

6. Hardin PE: Molecular genetic analysis of circadian timekeeping in Drosophila. Adv Genet 2011, 74:141-173.

7. Meireles-Filho AC, Rivas GBS, Gesto JS, Machado RC, Britto C, de Souza NA Peixoto AA: The biological clock of an hematophagous insect: locomotor activity rhythms, circadian expression and downregulation after a blood meal. FEBS Lett 2006, 580:2-8.

8. Jones MDR, Gubbins SJ: Changes in the circadian flight activity of the mosquito Anopheles gambiae in relation to insemination, feeding and oviposition. Physiol Entomol 1978, 3:213-220.

9. Jones MDR, Gubbins SJ: Modification of circadian flight activity in the mosquito Anopheles gambiae after insemination. Nature 1977, 268:731-732.

10. Hawkes F, Young S, Gibson G: Modification of spontaneous activity patterns in the malaria vector Anopheles gambiae sensu stricto when presented with host-associated stimuli. Physiol Entomol 2012, 37:233-240.

11. Saunders DS: Insect Clocks. Boston: Amsterdam Elsevier; 2002.

12. Forattini OP, Kakitani I, dos Santos RL, Kobayashi KM, Ueno HM, Fernandez Z: Adult Aedes albopictus and Ae. scapularis behaviour (Diptera: Culicidae in Southeastern Brazil. Rev Saude Publica 2000, 34:461-467.

13. Macdonald WW: Aedes aegypti in Malaya. II- Larval and adult biology. Ann Trop Med Parasitol 1956, 50:399-414.

14. Del Rosario A: Studies on the biology of Philippine mosquitoes. II. Observations on the life and behaviour of Aedes albopictus (Skuse) in the laboratory. Phil J Sci 1963, 92:89-103.

15. Mori A, Wada Y: The gonotrophic cycle of Aedes albopictus in the field. Trop Med 1977, 19:141-146.

16. Klowden MJ: Endogenous factors regulating mosquito host-seeking behaviour. Ciba Found Sym 1996, 200:212-225.

17. Klowden MJ: The check is in the male: male mosquitoes affect female physiology and behaviour. J Am Mosq Control Assoc 1999, 15:213-220.

18. Jones MDR: The programming of circadian flight activity in relation to mating and the gonotrophic cycle in the mosquito Aedes aegypti. Physiol Entomol 1981, 6:307-313.

19. Clements AN: Adult circadian rhythms. In Sensory reception and behaviour Eastbourne, UK: CABI publishing; 1999:206-238.

20. Filipecki AT, Machado CJ, Valle S, Teixeira MO: The Brazilian legal framework on the scientific use of animals. ILAR J 2011, 52:E8-E15.

21. Rosato $E$, Kyriacou CP: Analysis of locomotor activity rhythms in Drosophila. Nat Protoc 2006, 1:559-568.
22. Gentile C, Meireles-Filho AC, Britto C, Lima JB, Valle D, Peixoto AA: Cloning and daily expression of the timeless gene in Aedes aegypti (Diptera: Culicidae). Insect Biochem Mol Biol 2006, 36:878-884

23. Gentile C, Rivas GBS, Meireles-Filho AC, Lima JB, Peixoto AA: Circadian expression of clock genes in two mosquito disease vectors: cry2 is different. J Biol Rhythms 2009, 24:444-451.

24. Lima-Camara TN, Bruno RV, Luz PM, Castro MG, Lourenço-de-Oliveira R, Sorgine MHF, Peixoto AA: Dengue infection increases the locomotor activity of Aedes aegypti females. PLoS One 2011, 6:e17690

25. Williams CB: The use of logarithms in the interpretation of certain entomological problems. Ann Appl Biol 1937, 24:404-414.

26. Das S, Dimopoulos G: Molecular analysis of photic inhibition of blood- feeding in Anopheles gambiae. BMC Physiol 2008, 8:23.

27. Rund SS, Hou TY, Ward SM, Collins FH, Duffield GE: Genome-wide profiling of diel and circadian gene expression in the malaria vector Anopheles gambiae. Proc Natl Acad Sci U S A 2011, 108:E421-E430.

28. Taylor B, Jones MDR: The circadian rhythm of flight activity in the mosquito Aedes aegypti (L.): the phase-setting effects of light-on and light-off. J Exp Biol 1969, 51:59-70.

29. Yee WL, Foster WA: Diel sugar-feeding and host-seeking rhythms in mosquitoes (Diptera: Culicidae) under laboratory conditions. J Med Entomol 1992, 29:784-791.

30. Brito LP, Linss JG, Lima-Camara TN, Belinato TA, Peixoto AA, Lima JB, Valle D, Martins AJ: Assessing the effects of Aedes aegypti kdr mutations on pyrethroid resistance and its fitness cost. PLoS One 2013, 8:e60878.

31. Lima-Camara TN, Codeço CT, Honório NA, Bruno RV, Peixoto AA, Lounibos LP: Male accessory gland substances from Aedes albopictus affect the locomotor activity of Aedes aegypti females. Mem Inst Oswaldo Cruz 2013, 108(Suppl. I):18-25.

32. Rowland M: Changes in the circadian flight activity of the mosquito Anopheles stephensi associated with insemination, blood-feeding, oviposition and nocturnal light intensity. Physiol Entomol 1989, 14:77-84.

33. Gentile C, Rivas GBS, Lima JB, Bruno RV, Peixoto AA: Circadian clock of Aedes aegypti: effects of blood-feeding, insemination and RNA interference. Mem Inst Oswaldo Cruz 2013, 108(Suppl. I):80-87.

34. Klowden MJ, Lea AO: Blood meal size as a factor affecting continued host-seeking by Aedes aegypti (L.). Am J Trop Med Hyg 1978, 27:827-831.

35. Klowden MJ, Lea AO: Abdominal distention terminates subsequent host-seeking behaviour of Aedes aegypti following a blood meal. J Insect Physiol 1979, 25:583-585

36. Lima-Camara TN, Honório NA, Lourenço-de-Oliveira R: Parity and ovarian development of Aedes aegypti and Ae. albopictus (Diptera: Culicidae) in metropolitan Rio de Janeiro. J Vector Ecol 2007, 32:34-40.

37. Lima-Camara TN: Activity patterns of Aedes aegypti and Aedes albopictus (Diptera: Culicidae) under natural and artificial conditions. Oecologia Australis 2010, 14:737-744.

doi:10.1186/1756-3305-7-304

Cite this article as: Lima-Camara et al:: Effects of insemination and blood-feeding on locomotor activity of Aedes albopictus and Aedes aegypti (Diptera: Culicidae) females under laboratory conditions. Parasites \& Vectors 2014 7:304

\section{Submit your next manuscript to BioMed Central and take full advantage of:}

- Convenient online submission

- Thorough peer review

- No space constraints or color figure charges

- Immediate publication on acceptance

- Inclusion in PubMed, CAS, Scopus and Google Scholar

- Research which is freely available for redistribution 